\title{
Decontamination Efficiency of Phenylethylene by an Activated Carbon-Based Adsorbent
}

\author{
Wang Shouzhong, Zhou Zhen, Zhang Tong †, Fang Xiaojun and Miao Chaoyang \\ *State Key Laboratory of Technologies in Space Cryogenic Propellants, Beijing Special Engineering Design and \\ Research Institute, Beijing 100028, China \\ †Corresponding author: Zhang Tong; 1164177746@qq.com
}

\author{
Nat. Env. \& Poll. Tech. \\ Website: www.neptjournal.com \\ Received: 08-11-2019 \\ Revised: $25-11-2019$ \\ Accepted: $16-01-2020$ \\ Key Words: \\ Phenylethylene \\ Activated carbon \\ Oxidizing agent \\ Decontamination efficiency
}

\begin{abstract}
A sudden leak of phenylethylene is an urgent issue for the surrounding environment. To mitigate its negative effect, the decontamination efficiency of phenylethylene on an activated carbon (AC)-based adsorbent was investigated. Factors such as the particle size and the temperature, that could affect the adsorption ratio, were explored. Meanwhile, the efficiency of $\mathrm{AC}$, pretreated with different $\mathrm{KMnO}_{4}$ and $\mathrm{NaClO}$ concentrations, was examined. It was proven that the decontamination efficiency was higher for the 300-mesh AC compared to the 200-mesh AC. The introduction of the oxidizer, $\mathrm{KMnO}_{4}$, had a negative effect on phenylethylene adsorption. Nevertheless, the $\mathrm{NaClO}$-modified AC showed a positive influence on phenylethylene removal, while its decontamination gradually improved with the increase of the $\mathrm{NaClO}$ concentration. It was also found that the adsorption rate of phenylethylene was ascended with the temperature rise. After $1 \mathrm{~h}$ of adsorption with $\mathrm{AC}$ heated to $200^{\circ} \mathrm{C}$, no phenylethylene desorption was observed.
\end{abstract}

\section{INTRODUCTION}

Phenylethylene $\left(\mathrm{C}_{8} \mathrm{H}_{8}\right)$ is a typical volatile organic compound (VOC) (Ren et al. 2013), which is mephitic, toxic and flammable. Phenylethylene is an important organic chemical and is widely used in the production of synthetic resin, synthetic rubber, and ion-exchange resin. Its strong irritation and the narcotic effect would greatly harm people's respiratory tracts and eyes. In recent years, several phenylethylene leakage accidents have occurred in China (Yu 2012, Zhou et al. 2016), such as the phenylethylene leakage at the Yangtze River in 2001, the phenylethylene leakage due to tank car turnover in Lueyang City in 2010, and the phenylethylene spill at Jiangyin Section of the Yangtze River in 2011.

In case of emergency, the firefighters shall take simple and efficient measures to decontaminate hazardous chemicals quickly and conveniently. The decontamination materials should apply to the existing equipment and have a wide source, low cost, large output, and high adsorption efficiency (Jiang et al. 2017, Wang et al. 2016). Decontamination generally consists of chemical methods and physical methods. In particular, the physical methods include flushing, adsorption, evaporation, and reverse osmosis, while the chemical methods include neutralization, catalysis and oxidation (Li et al. 2014, Liu et al. 2010, Yao et al. 2016, Zhou et al. 2017).

Regarding the adsorption method, the decontamination is achieved by using a certain adsorbent to concentrate the molecular pollutants on its surface or in the internal pores (Yu et al. 2019, Zhou et al. 2019, Zhou et al. 2015). The use of large Brunauer, Emmett and Teller (BET) and abundant micropores resulted in the adsorption capacity of AC up to $30-80 \mathrm{mg} \cdot \mathrm{g}^{-1}$ (Guo et al. 2010), employing mainly granular, powdered, and fibrous AC. In 2008 Pan et al. (2008) studied the adsorption of ammonia-modified $\mathrm{AC}$ and determined the porous structure and surface acid-base groups of several modified AC fibres, while the effect of the porous structure and the surface groups on the adsorption of phenylethylene was also discussed. Results showed that, after ammonia modification, the basic group content, the pore volume, and the BET features of the AC fibre surface increased, thus further improving the adsorption capacities of AC fibre on phenylethylene. In the study by Ren et al. (2013), AC and its modification were initially prepared from municipal sludge. The BET, acid-base group content, phenylethylene static adsorption, and desorption activation energy of AC were then compared before and after modification. It was demonstrated that the BET of sludge AC, modified by $0.1 \mathrm{M}$ copper sulphate solution, was increased by $32 \%\left(705 \mathrm{~m}^{2} \cdot \mathrm{g}^{-1}\right)$ compared to the value prior to modification. The impregnation of the aluminium sulphate and copper sulphate solutions increased the surface acid group content of sludge AC, while the surface basic group content was reduced. Besides, the adsorption capacity of phenylethylene was increased more than two times compared to the precursor (211.4 and 178.8 
mg. $\mathrm{g}^{-1}$, respectively). The adsorption penetration time for phenylethylene was increased from 2 to $10 \mathrm{~min}$ and the desorption activation energy was extended from 2.94 to $>6.00$ kJ.mol ${ }^{-1}$. Instead, Li et al. (2014) degraded phenylethylene by ozonation. Specifically, phenylethylene was effectively decontaminated by the ozone-coupled biological AC, whereas the decontamination rates of 1,2 , and $4 \mathrm{ppm}$ phenylethylene were $100 \%, 60.6 \%$ and $57.9 \%$, respectively.

The current study aimed to explore the decontamination behaviour of phenylethylene via $\mathrm{AC}$ in case of sudden leakage. The effect of particle size, thermal treatment, and oxidant modification on the phenylethylene adsorption on $\mathrm{AC}$ was investigated. Meanwhile, the second-order kinetic equation was used to illustrate the adsorption behaviour. The results of our investigation shed new light on the emergency disposal of sudden phenylethylene leakage.

\section{MATERIALS AND METHODS}

\section{Reagents and Apparatus}

Phenylethylene was procured from Tianjin Tianli Chemical Reagent (China). $\mathrm{KMnO}_{4}$ and $\mathrm{NaClO}$ were obtained from Sinopharm Chemical Reagent (China). The abovementioned reagents were of analytical grade and dissolved in deionized water (18.2 M , Milli-Q, USA). AC was purchased from Jiangshan Lvyi Bamboo Charcoal (China) and was used after following purification process. After grinding and sieving, the AC was cleaned with deionized water before boiling. Afterwards, the solution was continuously stirred for $1 \mathrm{~h}$ and repeatedly flushed with deionized water to remove the surface impurity. Finally, the AC was dried for $12 \mathrm{~h}$ in a drying oven at $80^{\circ} \mathrm{C}$. After being tested with the BET detector (ASAP 2460, Micromeritics, USA), the specific surface area of the adsorbent reached $873.1 \mathrm{~m}^{2} \cdot \mathrm{g}^{-1}$ (Fig. 1).

Fig. 2 shows a self-made apparatus that was used during the current study. It mainly consisted of four parts, namely a decontamination chamber, a water treatment agent atomization injection system, a dry powder treatment agent injection system, and a foam injection system. The decontamination chamber consisted of a tank-8 $(700 \mathrm{~mm} \times 700 \mathrm{~mm} \times 800$ $\mathrm{mm}$ ), an oil evaporating pan-21 (120 $\mathrm{mm}$ high), an air sac-7 (for balancing the chamber pressure during jet decontamination), a steam sampling tube-20, a filter-12, and a vapour concentration analyzer-13 (CEM200 Multi-Gas Emergency Detector, Beijing Taihua Hengyue Technology Development Co., Ltd.). The water treatment agent atomization injection system consisted of a sink-1, a liquid pump-2, a transfer tube16 , and a spray nozzle- 3 . The dry powder treatment agent injection system consisted of an air pump-4, a dry powder tank-5, a dry powder nozzle-6, a dry powder tube-18, and a compressed air tube-19. The foam injection system consisted of a foam concentrate tank-11, a foam concentrate tube-17, a foam pump-10, and a foam nozzle-9.

\section{Experimental Steps}

1. After treating the dry powder tank-5 with vacuum, it was added in the tank, and the pressure was adjusted to 8 bar. Then, the nozzle above the experimental tank was connected to the dry powder tank. Boiling water of $50 \mathrm{~mm}$ in height was added to the liquid tray-21 and $15 \mathrm{~mL}$ of phenylethylene were sprayed on the surface of the boiling water to accelerate the evaporation.

2. The real-time monitoring was performed on the concentration of phenylethylene gas with the gas detector- 13 . When phenylethylene was substantially evaporated and

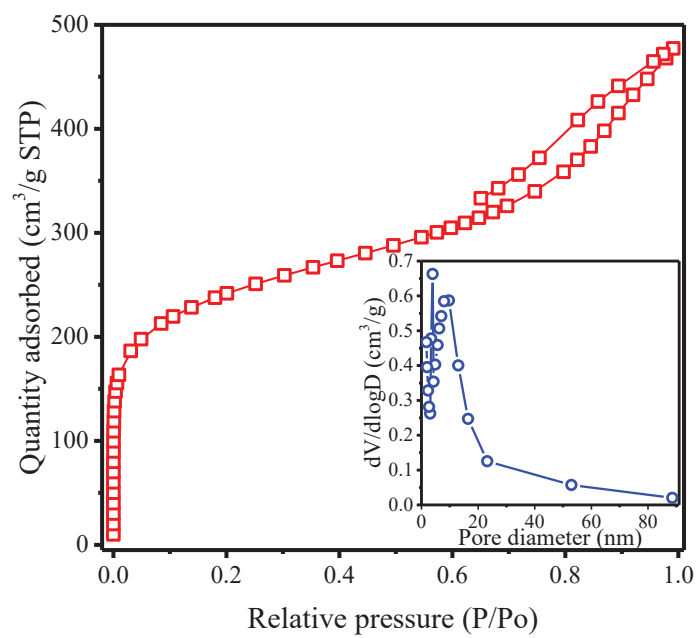

Fig. 1: Nitrogen adsorption-desorption isotherms and pore size distribution profiles (inset) of AC. 
the concentration reached a constant level, the valve of the dry powder tank was opened to spray the dry powder.

3. The spraying was repeated 10 times, each of which lasted for about $2 \mathrm{~s}$, and was completed in $3 \mathrm{~min}$. The change in phenylethylene concentration was continuously monitored and when the concentration dropped to a constant level, the experiment was considered to be complete.

\section{RESULTS AND DISCUSSION}

Effect of particle size: The 200 and 300-mesh AC are commonly used in the market and are applicable for firefighting equipment (Sun et al. 2017, Zhang et al. 2015). The 200 and 300 -mesh AC powders were selected for this study as well to compare their effect on the phenylethylene decontamination. During the experiment, all the experimental conditions, including the amount of $\mathrm{AC}$, the pressure of the dry powder tank, and the volatilization concentration of phenylethylene, were maintained constant. The real-time decontamination effect within five minutes is illustrated in Fig. 3.

The adsorption kinetics by the AC complied with the second-order kinetics, with insets showing the constants
$\left(\mathrm{R}^{2}>0.97\right)$, indicating that the chemical adsorption is the rate-limiting step of the decontamination process (Liang et al. 2018, Yan et al. 2017). The decontamination rate of the 300-mesh AC was $0.0127 \mathrm{~h}^{-1}$, i.e. about 1.25 times higher than the decontamination rate of the 200-mesh AC $(0.0102$ $\mathrm{h}^{-1}$ ). Moreover, the 300-mesh AC had a lower phenylethylene residual rate $(0.21$ vs. 0.26$)$, which indicated that its decontamination capability was greater than that of the 200-mesh AC. The 300-mesh AC had also smaller particle size, higher BET, and better hovering property, leading to improved phenylethylene elimination (Yu et al. 2017, Zhou et al. 2018).

Effect of oxidant modification: Fang et al. (2017) and Hu et al. (2017) documented that the oxidant impregnation could improve the adsorption capacity of the adsorbent. Based on these data, the 300-mesh AC was impregnated with a $\mathrm{KMnO}_{4}$ solution and a $\mathrm{NaClO}$ solution and then used for the decontamination of phenylethylene. Fig. 4 presents the adsorption kinetics of phenylethylene using the $\mathrm{AC}$ impregnated with 1.5 g. $\mathrm{L}^{-1}$ and 3.0 g. $\mathrm{L}^{-1}$ of the $\mathrm{KMnO}_{4}$ solution. The kinetics followed the second-order kinetic equation $\left(\mathrm{R}^{2}>0.99\right)$, which demonstrated that the impregnation with $\mathrm{KMnO}_{4}$ did not change the type of the phenylethylene adsorption kinetics. The decontamination process was still dominated by the

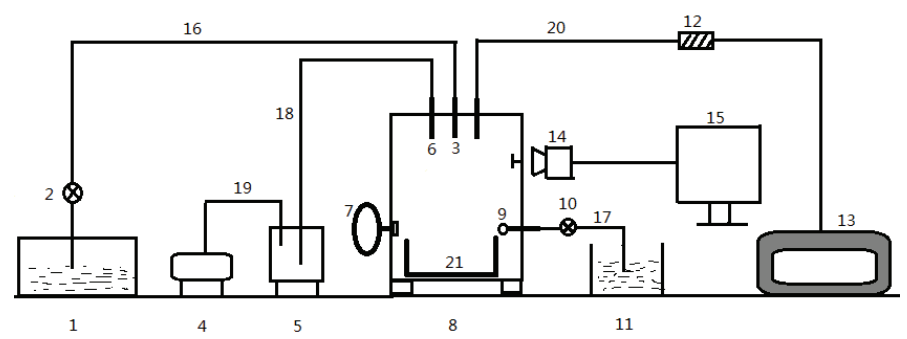

Fig. 2: Schematic diagram of the experiment.

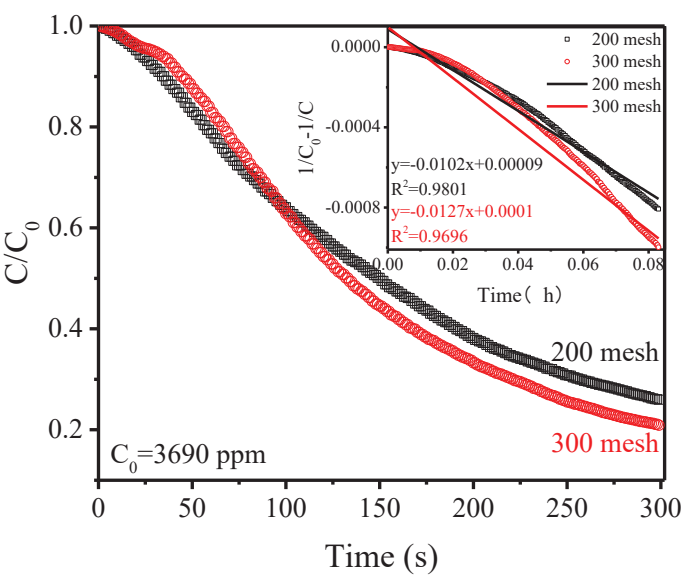

Fig. 3: Decontamination kinetics of phenylethylene on AC at different mesh number. The inset is the corresponding parameters of the second-order model. 
chemical adsorption. It was proven that the decontamination efficiency of phenylethylene was the same when using AC impregnated with 1.5 g. $\mathrm{L}^{-1}$ of $\mathrm{KMnO}_{4}\left(0.0117 \mathrm{~h}^{-1}\right)$ and 3.0 g. $\mathrm{L}^{-1}$ of $\mathrm{KMnO}_{4}\left(0.0116 \mathrm{~h}^{-1}\right)$. Whereas, both of the impregnated $\mathrm{AC}$ manifested the inferior adsorption rate than their pristine predecessor $\left(0.0127 \mathrm{~h}^{-1}\right)$, demonstrating that impregnation with $\mathrm{KMnO}_{4}$ negatively affected the phenylethylene adsorption. The effect of the oxidation treatment on $\mathrm{AC}$ had two sides. Although the oxidant impregnation increased the number of the surface hydroxyl, carboxyl, and carbonyl groups, hence improving the decontamination efficiency, the oxidant could etch the surface and pore walls of AC and occupy partial pores. Such effects could concomitantly decrease the BET and the AC pore volume and reduce the adsorption sites (Fang et al. 2017, Zhou et al. 2016a, 2016b). In this study, $\mathrm{KMnO}_{4}$ corroded the surface and pore walls of $\mathrm{AC}$ to such an extent that the negative impact predominated the positive effect.

According to previous reports, strong oxidants, such as nitric acid, easily damaged the pore walls, reduced the number of micropores, increased the number of mesopores and macropores, and reduced the BET, and the pore volume. Subsequently, they affected the adsorption efficiency (Gil \& Grange 1997). Other studies (Wang et al. 2012) reported that the modification of $\mathrm{AC}$ with $\mathrm{NaClO}$ was relatively mild, indicating that the pore structure was slightly damaged, while the number of phenolic hydroxyl, carboxyl, ketonic, and ether surface oxygen-containing functional groups was increased (Rivera-Utrilla et al. 2011).

Hence, $\mathrm{NaClO}$ solutions with $5 \%$ effective chlorine and $10 \%$ effective chlorine were selected to treat $\mathrm{AC}$, to carry out

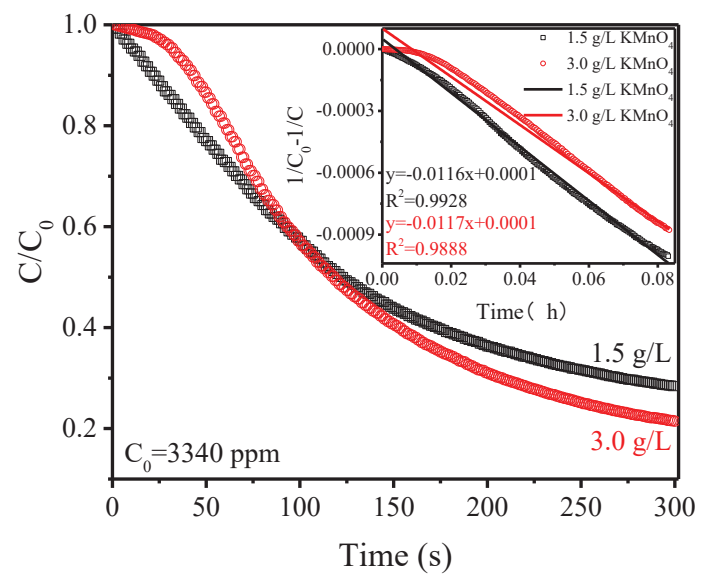

Fig. 4: Decontamination kinetics of phenylethylene on AC after 1.5 and $3.0 \mathrm{~g} / \mathrm{L} \mathrm{KMnO}_{4}$ immersion. The inset is the corresponding parameters of the second-order model. the decontamination test. Fig. 5 describes that the adsorption rate of $5 \% \mathrm{NaClO}$ impregnated $\mathrm{AC}$ was significantly elevated from 0.0127 to $0.0200 \mathrm{~h}^{-1}$, and further increased to 0.0217 $\mathrm{h}^{-1}$ with the increase of $\mathrm{NaClO}$ concentration. This improvement substantiated that the impregnation with $\mathrm{NaClO}$ could gradually increase the active sites on the surface of $\mathrm{AC}$ and in turn the decontamination efficiency of phenylethylene.

Effect of thermal modification: Thermal treatment is an easily achievable physical method for controlling the surface functional groups and pore structures of AC (Fang et al. 2017). Herein, we investigated the decontamination efficiency of phenylethylene using $\mathrm{AC}$ treated at $100^{\circ} \mathrm{C}$ and $200^{\circ} \mathrm{C}$ and the obtained results are displayed in Fig. 6 . The decontamination efficiency of phenylethylene after thermal modification was higher than that of pristine $\mathrm{AC}\left(0.0127 \mathrm{~h}^{-1}\right)$, while their decontamination efficiency gradually elevated with rising temperature $\left(0.0175-0.0190 \mathrm{~h}^{-1}\right)$. The adsorption capacity of the adsorbent for a specific adsorbate was mainly determined by physical properties such as BET, micropore volume, and pore size distribution, as well as its chemical properties such as the surface groups (Zhen et al. 2017, Zhen et al. 2016). The high-temperature activation improved the lattice structure of the AC, removed parts of impurities adsorbed on the surface and in the pores, and also increased the number of micropores or mesopores, thus increasing the BET of AC. Meanwhile, the high-temperature activation also oxidized some functional groups on the surface of AC, released a number of oxygen-containing functional groups, and enhanced the hydrogen bonding force, thus improving the adsorption efficiency (Fang et al. 2017).

Stability test: The adsorbed adsorbates may desorb over a

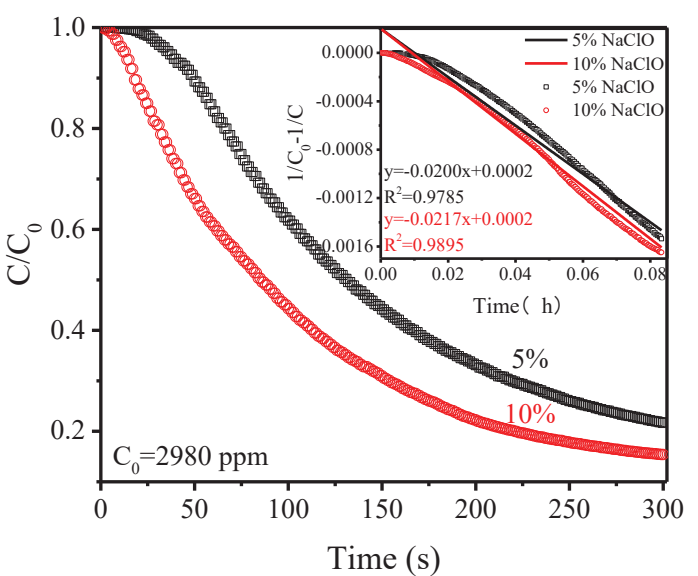

Fig. 5: Decontamination kinetics of phenylethylene on AC after $5 \%$ and $10 \% \mathrm{NaClO}$ immersion. The inset is the corresponding parameters of the second-order model. 


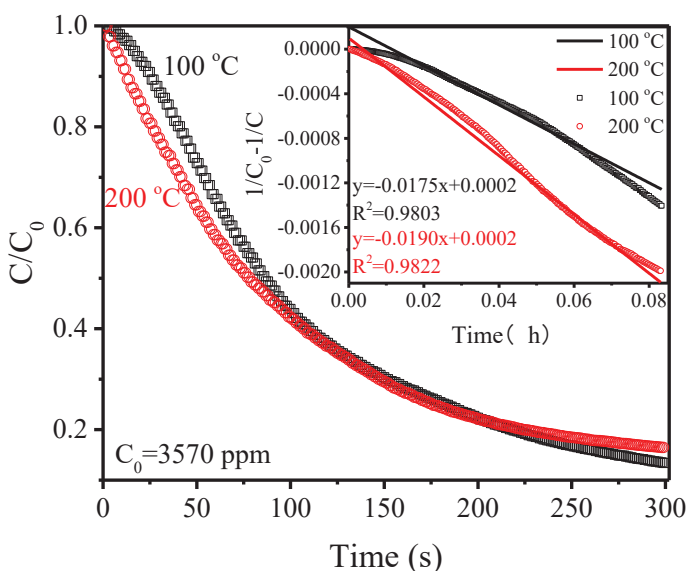

Fig. 6: Decontamination kinetics of phenylethylene on AC after 100 and $200^{\circ} \mathrm{C}$ heat treatment. The inset is the corresponding parameters of the second-order model.

long time (Zhou et al. 2016a, Zhou et al. 2017). The $200^{\circ} \mathrm{C}$ treated AC was selected to decontaminate phenylethylene and test its adsorption stability on AC. Fig. 7 illustrates the content of phenylethylene with the concomitant increase of the adsorption time, where an apparent decrease could be observed at a rudimentary stage spanning between 0-400 s. Subsequently, an equilibrium period was achieved. Specifically, the concentration of the phenylethylene gas in the decontamination chamber did not significantly rise within $1 \mathrm{~h}$, indicating that the phenylethylene molecule was tightly adsorbed on AC and was hard to be desorbed.

\section{CONCLUSIONS}

Herein, the effect of the particle size, the oxidant modification, and the thermal treatment on the decontamination efficiency of phenylethylene was studied. The AC with a higher mesh number resulted in a greater decontamination efficiency. The modification of $\mathrm{AC}$ with $\mathrm{KMnO}_{4}$ decreased the efficiency, while it was increased with the $\mathrm{NaClO}$ modification and was further elevated with the increase of the $\mathrm{NaClO}$ concentration. Thermal treatment enhanced the adsorption rate of phenylethylene, which escalated with the temperature rise. No phenylethylene desorption was detected $1 \mathrm{~h}$ after the $\mathrm{AC}$ adsorption.

\section{ACKNOWLEDGEMENTS}

The work was subsidized by the National Science \& Technology Pillar Program of China (2015BAK37B00).

\section{REFERENCES}

Fang, H., Li, Z., Ma, D. and Dong, H.. 2017. Research progress in modification of activated carbon as adsorbent used in flue gas desulfurization.

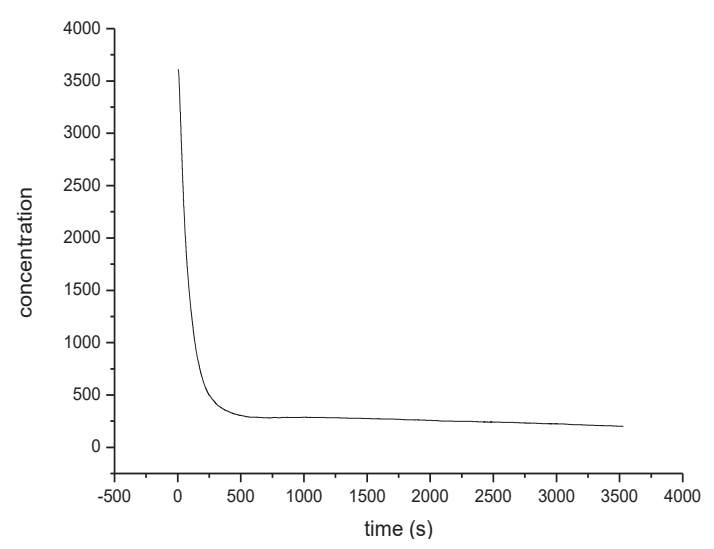

Fig. 7: Adsorption stability of phenylethylene on AC.

Energy for Metallurgical Industry, 36(6): 56-61.

Gil, A., Puente, G. D. L. and Grange, P. 1997. Evidence of textural modifications of an activated carbon on liquid-phase oxidation treatments. Microporous Materials, 12(1):51-61

Guo, C., Zhao, P. and Xiao, J. 2010. Application of several absorbing material to treat oil wastewater. Environmental Science and Management, 35(3): 96-98,102.

Hu, C., Lu, R., Huang, J. and Lv, X. 2017. Epoxidation of styrene by novel asymmetric Schiff base copper complex. Chemical Engineering, 45(10): 62-66.

Jiang, T., Ni, X., Wang, X. and Dong, C. 2017. A comparative study on the performances of multi-type agents used for emergency disposal of leaked methanol. Industrial Safety and Environmental Protection, 43(5): 62-65.

Li, T., Wang, Z., Bai, X. and Zhu, Y. 2014. Emergency treatment of styrene contamination in source water for waterworks. China Water \& Wastewater, 30(13): 6-9,14.

Liang, Q., Luo, H., Geng, J. and Chen, J. 2018. Facile one-pot preparation of nitrogen-doped ultra-light graphene oxide aerogel and its prominent adsorption performance of $\mathrm{Cr}(\mathrm{VI})$. Chemical Engineering Journal, 338: 62-71.

Liu, J., Ma, W., Wang, M. and Huang, L. 2010. Cleaning of waste gas containing styrene using a fungal biofilter. Environmental Engineering, 28(05): 66-69,73.

Pan, H., Li, Z., Xia, Q. and Li, J. 2008. Adsorption of styrene on ammonia-treated activated carbon fibres. Journal of Functional Materials, 2: 324-327.

Ren, A., Fu, F., Qu, Y. and Guo, B. 2013. Adsorption properties of modified activated sludge carbon to styrene. Environmental Chemistry, 32(5): 833-838.

Rivera-Utrilla, J., Sánchez-Polo, M., Gómez-Serrano, V., Álvarez, P. M., Alvim-Ferraz, M. C. M. and Dias, J. M. 2011. Activated carbon modifications to enhance its water treatment applications. An overview. Journal of Hazardous Materials, 187(1): 1-23.

Sun, Z., Ding, Y., Geng, Z. and Gao, J. 2017. Comparison of adsorption properties of biomass based activated carbon for p-nitrophenol. Industrial Catalysis, 25(6): 74-77.

Wang, K. and Xu, Q. 2012. Effect of several different treatment methods on surface chemical properties of activated carbon. Chinese Journal of Environmental Engineering, 6(2): 373-380.

Wang, S., Dong, C., Ni, X. and Cong, H. 2016. Experimental study on effectiveness of composite dry powder in emergence disposal of leaked methanol. China Safety Science Journal, 26(5): 164-168. 
Yan, L., Tu, H., Chan, T. and Jing, C. 2017. Mechanistic study of simultaneous arsenic and fluoride removal using granular $\mathrm{TiO}_{2}$-La adsorbent. Chemical Engineering Journal, 313: 983-992.

Yao, J., Zhou, Z., Pang, Z. and Liu, B. 2016. Research progress of ultrasonic wave combined technology for water treatment. Contemporary Chemical Industry, 45(11): 2660-2665.

Yu, A. 2012. Establishing precautionary and emergency handling system for water pollution: Application in Nanjing chemical industrial park. Environmental Monitoring Management and Technology, 24(06): 6-10.

Yu, Y., Yan, L., Cheng, J. and Jing, C. 2017. Mechanistic insights into $\mathrm{TiO}_{2}$ thickness in $\mathrm{Fe}_{3} \mathrm{O}_{4} @ \mathrm{TiO}_{2}$-GO composites for enrofloxacin photodegradation. Chemical Engineering Journal, 325: 647-654.

Yu, Y. Q., Zhou, Z., Ding, Z. X., Zuo, M. M., Cheng, J. M. and Jing, C. Y. 2019. Simultaneous arsenic and fluoride removal using $\{201\}$ $\mathrm{TiO}_{2}-\mathrm{ZrO}_{2}$ : Fabrication, characterization, and mechanism. Journal of Hazardous Materials, 377: 267-273.

Zhang, B., Tang, X., Yi, H. and Wang, Z. 2015. Study on NO adsorptive removal on modified activated carbon. New Chemical Materials, 43(7): $111-113,121$

Zhen, Z., Jilun, Y., Xing, Z., Zhaoxia, D. and Meimei, Z. 2017. Enhanced effluent quality of ceramic membrane ultrafiltration combined with UV/ $\mathrm{TiO}_{2}$ photocatalysis. Nature Environment and Pollution Technology, 16(3): 695-702.

Zhen, Z., Yao, J., Pang, Z. and Bo, L. 2016. Optimization of electrocoagulation process to eliminate $\mathrm{COD}_{\mathrm{Mn}}$ in micro-polluted surface water using response surface method. Journal of Dispersion Science and Technology, 37(5): 743-751.
Zhou, Y., Li, J., Chen, F. and Ning, Y. 2016. Study on the 3D fluorescence feature of styrene and emergent treatment of styrene pollutant in water. Spectroscopy and Spectral Analysis, 36(7): 2169-2172.

Zhou, Z., Yao, J., Liu, B., Pang, Z. and Zhang, X. 2017. Research progress on water treatment by $\mathrm{TiO}_{2}$ photocatalysis. Chemistry \& Bioengineering, 34(4): 1-5,9.

Zhou, Z., Yao, J., Pang, Z. and Liu, B. 2015. Review of research development in electrocoagulation technology in water treatment. Water Purification Technology, 34(5): 9-15,38.

Zhou, Z., Yao, J., Pang, Z. and Liu, B. 2016a. Optimization of electrocoagulation-ceramic micro-filtration membrane process by response surface method. Environmental Pollution \& Control, 38(3): 39-44,49.

Zhou, Z., Yao, J., Pang, Z. and Liu, B. 2016b. Optimization of electrocoagulation process to eliminate $\mathrm{UV}_{254}$ in micro-polluted surface water using response surface method. Technology of Water Treatment, 42(3): $32-36,42$

Zhou, Z., Yao, J., Pang, Z., Liu, B. and Zhang, X. 2017. Effect of transmembrane pressure and crossflow velocity on membrane fouling using magnetic flocculation pretreatment. Environmental Engineering, 35(6): 10-14.

Zhou, Z., Yu, Y., Ding, Z., Zuo, M. and Jing, C. 2018. Modulating high-index facets on anatase $\mathrm{TiO}_{2}$. European Journal of Inorganic Chemistry, 2018(6): 683-693.

Zhou, Z., Yu, Y., Ding, Z., Zuo, M. and Jing, C. 2019. Competitive adsorption of arsenic and fluoride on $\left\{\begin{array}{lll}2 & 0 & 1\end{array}\right\} \mathrm{TiO}_{2}$. Applied Surface Science, 466: 425-432. 